\title{
Hipertrofia Ventricular Esquerda do Atleta. Resposta Adaptativa Fisiológica do Coração
}

\author{
Left Ventricular Hypertrophy of Athletes. Adaptative Physiologic Response of \\ the Heart
}

Nabil Ghorayeb, Michel Batlouni, Ibraim M. F. Pinto, Giuseppe S. Dioguardi

Instituto Dante Pazzanese de Cardiologia - São Paulo, SP

\section{Objetivo}

Verificar se a hipertrofia ventricular esquerda (HVE) de atletas competitivos de resistência (maratonistas) representa processo adaptativo, puramente fisiológico, ou se pode envolver aspectos patológicos em suas características anatômicas e funcionais.

\section{Métodos}

De novembro de 1999 a dezembro de 2000, foram separados consecutivamente de 30 maratonistas em atividade esportiva plena, idade inferior a 50 anos, com HVE, previamente documentada, e sem cardiopatia subjacente. Foram submetidos aos exames: clínico, eletrocardiograma, ecodopplercardiograma, e teste ergométrico (TE). Quinze foram sorteados para realizar, também, teste ergoespirométrico e ressonância magnética (RM) do coração.

\section{Resultados}

Nos TE, todos apresentavam boa capacidade física cardiopulmonar, sem evidências de resposta isquêmica ao exercício, sintomas ou arritmias. No ecodopplercardiograma, os valores do diâmetro e espessura diastólica da parede posterior do ventrículo esquerdo (VE), do septo interventricular, massa do VE e diâmetro do átrio esquerdo, foram significativamente maiores que os do grupo de não atletas, com idades e medidas antropométricas semelhantes. A média da massa do VE dos atletas indexada à superfície corpórea $(126 \mathrm{~g} / \mathrm{m} 2)$ foi significativamente maior que a do grupo controle $(70 \mathrm{~g} / \mathrm{m} 2)(\mathrm{p}<0,001)$. A RM mostrou que não havia prejuízo da força contrátil ou da performance ventricular esquerda e valores de volume diastólico final, volume sistólico final e fração de ejeção dentro dos limites da normalidade. Por outro lado, a massa do VE média, $162,93 \pm 17,90 \mathrm{~g}$, e a espessura parietal ventricular, $13,67 \pm 2,13$ $\mathrm{mm}$, ao final da diástole no grupo atleta, diferiu significativamente do controle: $110 \pm 14,2 \mathrm{~g}(\mathrm{p}=0,0001)$ e $8 \pm 0,9 \mathrm{~mm}$, respectivamente $(p=0,0001)$. 0 mesmo ocorreu na média da espessura ao final da sístole, que foi $18,87 \pm 3,40 \mathrm{~mm}$ (controle: $10 \pm 1,80 \mathrm{~mm}, \mathrm{p}=0,0001$ ).

\section{Conclusão}

Os resultados permitiram concluir que a HVE de maratonistas em período de atividade esportiva plena, avaliada por métodos não invasivos, representa resposta adaptativa ao treinamento físico intensivo e prolongado, com características fisiológicas.

\section{Palavras-chave}

hipertrofia cardíaca, coração de atleta, cardiomegalia do atleta

\section{ОвJECTIVE}

To verify whether left ventricular hypertrophy (LVH) of elite competition athletes (marathoners) represents a purely physiological, adaptative process, or it may involve pathological aspects in its anatomical and functional characteristics.

\section{Methods}

From November 1999 to December 2000, consecutive samples from 30 under 50-year-old marathoners in full sportive activity, with previously documented $\mathrm{LVH}$ and absence of cardiopathy were selected. They were submitted to clinical exams, electrocardiogram, color Doppler echocardiogram and exercise treadmill test (ETT). Fifteen were assorted to be also submitted to ergoespirometric test and heart magnetic resonance imaging (MRI).

\section{RESULTS}

In ETT, all of them showed good physical pulmonary capacity, with no evidences of ischemic response to exercise, symptoms or arrhythmias. In Doppler echocardiogram, values of diameter and diastolic thickness of LV posterior wall, interventricular septum, LV mass and left atrium diameter, were significantly higher when compared to non-athlete control group, with similar ages and anthropometric measurements. The mean of LV mass of athletes indexed to body surface $(126 \mathrm{~g} / \mathrm{m} 2)$ was significantly greater than the one in control group $(70 \mathrm{~g} / \mathrm{m} 2)(p<0.001)$. Magnetic resonance imaging (MRI) showed there was not impairment of contractile strength or LV performance, and values of end diastolic volume, end systolic volume and EF within limits of normality. On the other hand, average ventricular parietal mass, $162.93 \pm 17.90 \mathrm{~g}$, and LV parietal thickness, $13.67 \pm 2.13$ $\mathrm{mm}$, at the end of diastole in athlete group, differed significantly from control group: $110 \pm 14.2 \mathrm{~g}(p=0.0001)$ and $8 \pm 0.9 \mathrm{~mm}$, respectively $(p=0.0001)$. The same happened to the thickness at the end of systole, which was $18.87 \pm 3.40 \mathrm{~mm}$ (control group: $10 \pm 1.80 \mathrm{~mm}, p=0.0001$ ).

\section{Conclusion}

Results allowed for concluding that LVH in marathoners in full sportive activity period, assessed by non-invasive methods, represents an adaptative response to intensive and prolonged physical training, with purely physiological characteristics.

\section{KEY WORDS}

cardiac hypertrophy, athletic heart, athletic cardiomegaly

Correspondência: Nabil Ghorayeb・Rua Bom Pastor, $1163 \bullet 04203-051$ • São Paulo, SP 
A hipertrofia cardíaca do atleta altamente treinado foi reconhecida há mais de um século por Bergmann (1884) e Parrot (1893), citados por Rost ${ }^{1}$, e constitui um dos achados mais freqüentes do coração de atleta.

No entanto, controvérsias ainda persistem sobre seu real significado. Tratar-se-ia de processo puramente fisiológico, hipertrofia compensatória ou adaptativa, necessária para manter ótima performance cardíaca em condições de sobrecarga circulatória aumentada; ou envolveria o potencial de induzir, a longo prazo, alterações patológicas, relacionadas à estrutura miocárdica e ao comprometimento do desempenho cardíaco? A maioria dos autores apóia a primeira hipótese ${ }^{1-5}$. De outra parte, alguns autores têm questionado esse ponto de vista, admitindo que a hipertrofia ventricular esquerda do atleta possa ter conseqüências patológicas, considerando que, não raramente, os valores acentuadamente aumentados da espessura parietal e da dilatação ventricular se sobrepõem aos da miocardiopatia hipertrófica ou dilatada 6 .

0 treinamento físico intensivo e prolongado induz adaptações cardiovasculares que permitem ao coração do atleta desempenho físico excepcional ${ }^{7-9}$. Entretanto, essas adaptações incluem alterações funcionais e anatômicas, que podem situar-se fora dos limites da normalidade e cujo significado clínico e prognóstico têm sido objeto de intensa discussão e controvérsias ${ }^{1,6}$.

Vários e conhecidos são os efeitos cardiovasculares do treinamento físico vigoroso, praticado durante longos períodos, observados experimentalmente e em atletas competitivos, altamente treinados ${ }^{2,10,11}$. Verifica-se, ainda, maior eficiência mecânica da musculatura esquelética, aumento da capilarização, atividades enzímicas, aumento da capacidade funcional pulmonar e melhor relação ventilação/perfusão. Essas alterações cardiovasculares resultam de complexa interação de mecanismos centrais e periféricos, operando em níveis estruturais, eletrofisiológicos, bioquímicos, metabólicos e neurogênicos ${ }^{12}$. E dependem da intensidade e duração do treinamento, do tipo de atividade atlética e de fatores genéticos.

\section{MÉtodos}

No período de novembro de 1999 a dezembro de 2000, foram atendidos 306 atletas, rotineiramente encaminhados por suas respectivas entidades esportivas, para avaliação cardiológica pré-participação. Destes, foram separados, consecutivamente, 30 atletas, dezoito da raça branca, onze da negra e um, da amarela, que preencheram os critérios de inclusão: sexo masculino, com idade inferior a 50 anos (idades de 24 a 48 anos, média 37,5 $\pm 6,21$ ); praticantes de esporte aeróbio de alta resistência (maratonistas) há mais de três anos e em período de atividade atlética plena; hipertrofia ventricular esquerda ao ecocardiograma bidimensional com doppler colorido (espessura e massa ventricular esquerda acima dos limites da normalidade); ausência de doença cardiovascular subjacente. Dos 30 atletas, foram sorteados 15 para se submeterem ao exame de ressonância magnética do coração.

Seguiu-se o protocolo da Seção Médica de Cardiologia do Esporte do Instituto Dante Pazzanese de Cardiologia, do Estado de São Paulo: entrevista clínica, com especial atenção aos sintomas e sinais cardiovasculares, em repouso ou induzidos pelo esforço, à duração, intensidade e freqüência do treinamento, eletrocardiograma em repouso ${ }^{13}$, teste ergométrico em esteira rolante, usando-se protocolo de Bruce $^{14}$, até sintoma ou sinal limitante, e teste cardiopulmonar ou ergoespirométrico, com protocolo de rampa padronizado para atletas, nos 15 atletas sorteados para realizar ressonância magnética do coração. Os registros do ecocardiograma com Doppler colorido dos 30 atletas foram realizados em condições de repouso e sem a administração de fármacos. Cada exame constou de avaliações nos modos M, bidimensional e Doppler nas modalidades pulsado, contínuo e por mapeamento de fluxo a cores ${ }^{15,16}$.

Pela ressonância magnética (RM), foi quantificada a massa miocárdica, analisado o padrão de contração das fibras cardíacas e avaliada a função ventricular global e a contratilidade regional do ventrículo esquerdo pelo tagging ${ }^{17}$. Essa análise é subjetiva e determina se há ou não defeitos de contratilidade regional, comparando os corações dos atletas com os da população normal. A seqüência utilizada foi a cine-ressonância "FIESTA" com apnéia. As definições de bordas e a determinação dos volumes ventriculares foram feitas na própria estação de trabalho computadorizada, dedicada para esse fim, com programa próprio do fabricante.

Os resultados obtidos no grupo de atletas foram comparados com os observados em um grupo controle de 30 indivíduos normais, sedentários, com características antropométricas semelhantes.

\section{Resultados}

Nenhum dos 30 atletas apresentou histórico clínico de doenças familiares ou de morte súbita, nem antecedentes pessoais de importância clínica, e os exames físicos não mostraram anormalidades significativas.

A análise dos eletrocardiogramas (ECG) mostrou padrões comuns em atletas treinados: a) a freqüência cardíaca variou de 40 a 64 bpm, média 50,4 bpm $\pm 7,0$; b) bradicardia sinusal (menos de 60 bpm) em 26 atletas (87\%); c) bloqueio atrioventricular (BAV) de $1^{\circ}$ grau em cinco casos ( $17 \%$ ). Em um, BAV de $2^{\circ}$ grau, tipo Mobitz 1 intermitente; d) distúrbio de condução do ramo direito em 11 casos (37\%), um dos quais com bloqueio completo do ramo direito, intermitente ( $Q R S>12 \mathrm{~mm}$ ); e) sobrecarga atrial esquerda em seis casos (20\%); f) hipertrofia ventricular esquerda ${ }^{18}$ em oito casos (27\%); g) padrão de repolarização ventricular precoce em 19 casos (63\%), padrão juvenil, em cinco (17\%), ondas T negativas/achatadas (parede anterior), em dois (7\%). 


\begin{tabular}{|c|c|c|c|c|c|c|c|}
\hline & \multicolumn{2}{|c|}{$\mathrm{FC}$} & \multicolumn{2}{|c|}{$\mathrm{PA}$} & \multirow{2}{*}{$\begin{array}{c}T \\
\min \end{array}$} & \multirow{2}{*}{$\begin{array}{l}\mathrm{VO}_{2} \max \\
\mathrm{ml} / \mathrm{kg} / \mathrm{min}\end{array}$} & \multirow[t]{2}{*}{ MET } \\
\hline & Inicial & Final & Inicial & Final & & & \\
\hline Mín. & 36 & 163 & $110 \times 60$ & $170 \times 70$ & 10 & 46 & 14,6 \\
\hline Máx. & 67 & 197 & $140 \times 90$ & $220 \times 90$ & 21 & 77,3 & 22,4 \\
\hline Média & 55,7 & 179,9 & $127,7 \times 80,5$ & $191,5 \times 80,2$ & 12,6 & 63,64 & 19,1 \\
\hline $\mathrm{DP}$ & 7,3 & 10,3 & $10 \times 8,1$ & $14,2 \times 9,5$ & 2,6 & 10,38 & 2,83 \\
\hline
\end{tabular}

Tabela II - Valores mínimos, máximos, médias e desvios-padrão dos dados obtidos nos testes cardiopulmonares

\begin{tabular}{|c|c|c|c|c|c|c|c|c|c|c|}
\hline & \multicolumn{2}{|c|}{ FC } & \multicolumn{2}{|c|}{ PA } & \multirow{2}{*}{$\begin{array}{c}\mathrm{T} \\
\min \end{array}$} & \multirow{2}{*}{$\begin{array}{c}\mathrm{VO}_{2} \\
\mathrm{ml} / \mathrm{kg} / \mathrm{min}\end{array}$} & \multirow[t]{2}{*}{ MET } & \multirow{2}{*}{$\begin{array}{c}\mathrm{VO}_{2} \mathrm{LA} \\
\mathrm{ml} / \mathrm{kg} / \mathrm{min}\end{array}$} & \multirow[t]{2}{*}{$\%$ FC max } & \multirow{2}{*}{$\begin{array}{c}\% \text { FC max } \\
\text { LA }\end{array}$} \\
\hline & Inicial & Final & Inicial & Final & & & & & & \\
\hline Mín & 55 & 165 & $110 \times 80$ & $170 \times 70$ & $10: 44$ & 48,6 & 14 & 28,1 & 92,4 & 70,2 \\
\hline Máx & 67 & 197 & $140 \times 90$ & $210 \times 85$ & $12: 18$ & 52,1 & 15 & 38,6 & 107,7 & 89,8 \\
\hline Média & 61 & 178 & $128 \times 81$ & $190 \times 77$ & $11: 35$ & 51,3 & 14,6 & 32,4 & 99,2 & 79,4 \\
\hline DP & 4,3 & 11,5 & $11,7 \times 6,5$ & $12,6 \times 7,5$ & 0,03 & 1,33 & 0,42 & 4,3 & 5,5 & 6,6 \\
\hline
\end{tabular}

Os resultados obtidos nos 30 testes ergométricos são apresentados na tabela I e os resultados dos 15 testes cardiopulmonares na tabela II.

Nos testes ergométrico e cardiopulmonar, os parâmetros clínicos e eletrocardiográficos observados e registrados, durante e após os mesmos, foram considerados compatíveis com a normalidade, em nenhum caso houve resposta isquêmica ao exercício, nem foram observado sintomas clínicos, arritmias, comportamento anormal da freqüência cardíaca ou da função ventricular. 0 motivo da interrupção do exercício em todos os casos foi a exaustão física que, em geral, ocorreu próxima à $\mathrm{FC}$ máxima prevista. A capacidade cardiorrespiratória dos 30 atletas foi considerada de nível funcional "bem condicionado", superior às encontradas em sedentários normais ${ }^{19}$.

Ecodopplercardiograma - Foram obtidos valores absolutos e valores indexados à massa corpórea e comparados a grupo controle de 30 não atletas, com idades e medidas antropométricas semelhantes (tabs. III e IV).

As médias do diâmetro do átrio esquerdo, do diâmetro sistólico do VE, das espessuras diastólicas do septo interventricular e da parede posterior do VE, da massa ventricular, da fração de ejeção e da porcentagem de encurtamento foram significativamente maiores $(p<0,001)$ no grupo atleta em comparação com o grupo controle (tab. III).

Essas mesmas variáveis indexadas à superfície corpórea também foram significativamente maiores no grupo atleta (tab. IV).

Especificamente, a média da massa do ventrículo esquerdo (VE) dos atletas, indexada à superfície corpórea (126 g/m2), foi significativamente maior que a do grupo controle $(70 \mathrm{~g} / \mathrm{m} 2)(p<0,001)$. A média da função

\section{Tabela III - Principais variáveis ecocardiográficas nos grupos atleta e controle}

\begin{tabular}{|c|c|c|c|c|c|}
\hline \multirow[t]{2}{*}{ Variáveis ecocardiográficas } & \multicolumn{2}{|c|}{ Atletas $(n=30)$} & \multicolumn{2}{|c|}{ Controle $(n=30) P$} & \\
\hline & Média (dp) & Variação & Média (dp) & Variação & \\
\hline Diâmetro da aorta (mm) & $33 \pm 3$ & 26 a 38 & $32 \pm 4$ & 24 a 38 & NS \\
\hline Diâmetro átrio esquerdo & $39 \pm 4$ & 25 a 42 & $34 \pm 4$ & 24 a 40 & $<0,001$ \\
\hline Diâmetro diastólico VE & $54 \pm 5$ & 44 a 66 & $50 \pm 4$ & 40 a 56 & $<0,001$ \\
\hline Diâmetro sistólico VE & $32 \pm 4$ & 24 a 39 & $32 \pm 4$ & 26 a 37 & $<0,001$ \\
\hline Espessura diastólica do septo ventricular & $11,0 \pm 0,5$ & 10 a 12 & $7,6 \pm 0,7$ & 6 a 9 & $<0,001$ \\
\hline Espessura diastólica da parede posterior & $11,0 \pm 0,6$ & 10 a 12 & $7,6 \pm 0,7$ & 6 a 9 & $<0,001$ \\
\hline Massa ventricular esquerda (g) & $234 \pm 41$ & 156 a 294 & $134 \pm 27$ & 81 a 171 & $<0,001$ \\
\hline Fração de ejeção & $0,70 \pm 0,05$ & 0,61 a 0,79 & $0,60 \pm 0,05$ & 0,53 a 0,73 & $<0001$ \\
\hline \% D D (Fração de encurtamento) & $40 \pm 4$ & 33 a 49 & $36 \pm 4$ & 31 a 47 & $<0,001$ \\
\hline
\end{tabular}

Tabela IV - Médias das principais variáveis ecocardiográficas obtidas nos grupo atleta e de controle indexadas à superfície corpórea

$\begin{array}{lcc}\text { Variáveis ecocardiográficas } & \text { Atletas }(\mathrm{n}=30) & \text { Controle }(\mathrm{n}=30) \\ \text { Diâmetro da aorta }\left(\mathrm{mm} / \mathrm{m}^{2}\right) & 18 \pm 2 & 17 \pm 2 \\ \text { Diâmetro do átrio esquerdo }\left(\mathrm{mm} / \mathrm{m}^{2}\right) & 21 \pm 2 & 17 \pm 2 \\ \text { Diâmetro diastólico do VE }\left(\mathrm{mm} / \mathrm{m}^{2}\right) & 29 \pm 4 & 26 \pm 4 \\ \text { Diâmetro sistólico do VE }\left(\mathrm{mm} / \mathrm{m}^{2}\right) & 17 \pm 3 & 17 \pm 3 \\ \text { Espessura diastólica do septo ventricular }\left(\mathrm{mm} / \mathrm{m}^{2}\right) & 5,9 \pm 0,5 & 3,9 \pm 0,4 \\ \text { Espessura diastólica da parede posterior }\left(\mathrm{mm} / \mathrm{m}^{2}\right) & 5,7 \pm 0,5 & 3,9 \pm 0,4 \\ \text { Massa ventricular esquerda }\left(\mathrm{g} / \mathrm{m}^{2}\right) & 126 \pm 24 & 70 \pm 15<0,001\end{array}$


Tabela V - Valores mínimo, máximo, média e desvio-padrão do volume diastólico final (VdF), volume sistólico final (VsF), fração de ejeção (FE), espessura diastólica e sistólica da parede ventricular, no grupo de atletas

\begin{tabular}{|lcccccc} 
& Vdf $(\mathrm{ml})$ & Vsf $(\mathrm{ml})$ & FE $(\%)$ & Massa $(\mathrm{g})$ & Espessura diastólica $(\mathrm{mm})$ & Espessura sistólica $(\mathrm{mm})$ \\
\hline Mín. & 70 & 20 & 57 & 130 & 11 & 15 \\
\hline Máx. & 140 & 53 & 72 & 240 & 19 & 26 \\
\hline Média & 99,07 & 36,8 & 63,67 & 162,93 & 13,67 & 18,87 \\
\hline DP & 20,09 & 7,97 & 4,53 & 17,90 & 2,13 & 3,40 \\
\hline
\end{tabular}

Tabela VI - Valores mínimo, máximo, média e desvio-padrão, do volume diastólico final (VdF), volume sistólico final (VsF), fração de ejeção (FE), espessura diastólica e sistólica da parede ventricular, no grupo controle

\begin{tabular}{lcccccc} 
& Vdf $(\mathrm{ml})$ & Vsf $(\mathrm{ml})$ & $\mathrm{FE}(\%)$ & Massa $(\mathrm{g})$ & Espessura diastólica $(\mathrm{mm})$ & Espessura sistólica $(\mathrm{mm})$ \\
\hline Mín, & 85 & 18 & 56 & 90 & 7 & 9 \\
\hline Máx, & 135 & 42 & 75 & 135 & 11 & 11 \\
\hline Média & 130,00 & 30,00 & 60,00 & 110,00 & 8,00 & 10,00 \\
DP & 15,00 & 5,45 & 8,20 & 14,20 & 0,90 & 1,80
\end{tabular}

ventricular esquerda, avaliada pela fração de ejeção, foi também significativamente maior no grupo atleta $(0,70)$ em comparação com a do controle $(0,60)(p<0,001)$. As variáveis da função diastólica do VE foram normais em todos os atletas.

Ressonância magnética - Os resultados nos 15 atletas mostraram valores de volume diastólico final, volume sistólico final e fração de ejeção dentro dos limites da normalidade.

A comparação dos resultados encontrados nos atletas com os valores dos 30 controles normais (tabs. $\mathrm{V}$ e $\mathrm{VI}$ ) não revelou diferença significativa quanto ao volume diastólico final $(p=0,7)$, ao volume sistólico final $(p=0,14)$ e à fração de ejeção $(p=0,20)$. Por outro lado, houve diferença significativa na massa ventricular esquerda e espessuras ventriculares, ao final da diástole e sístole. Assim, a massa média definida pela ressonância magnética foi $162,93 \pm 17,90$ g no grupo atleta versus $110 \pm 14,2$ g, no grupo controle $(p=0,0001)$; a média da espessura ao final da diástole foi $13,67 \pm 2,13 \mathrm{~mm}$ no grupo atleta e $8 \pm 0,9 \mathrm{~mm}$, no grupo controle $(p=0,0001)$; e a média da espessura ao final da sístole foi $18,87 \pm 3,40 \mathrm{~mm}$ nos atletas versus $10 \pm 1,80 \mathrm{~mm}$, no controle $(p=0,0001)$.

A despeito da magnitude das alterações encontradas, não havia dissinergia em nenhum caso, nem prejuízo da performance ventricular, o que foi atestado não apenas pela quantificação da fração de ejeção global, mas também pelo fato de a movimentação regional ventricular esquerda, analisada pelo tagging, não revelar alterações significativas em nenhum dos casos. A mensuração do espessamento regional de todos os atletas avaliados encontrar-se dentro dos limites da normalidade e a função diastólica dos atletas mostrar-se, igualmente, normal com discretas modificações, sem significado clínico (tab. VII).

Tabela VII - Análise da contratilidade segmentar do coração (avaliada pelo tagging), do espessamento regional e da função diastólica de 15 atletas

\begin{tabular}{lccc} 
& Espessamento regional & Tagging & Função diastólica \\
\hline Normal & 15 & 13 & 11 \\
$\begin{array}{l}\text { Alteração não } \\
\text { significativa }\end{array}$ & - & 02 & 04 \\
\hline
\end{tabular}

\section{Discussão}

O coração do atleta apresenta uma variedade de alterações morfológicas e funcionais, resultantes do treinamento físico rigoroso e sistemático, para melhorar a função do coração como bomba e a capacidade do sistema cardiovascular fornecer oxigênio aos músculos que se exercitam. As adaptações predominantes incluem: aumento da dimensão diastólica final da cavidade ventricular esquerda, da espessura parietal e da massa ventricular esquerda, melhora do enchimento diastólico e redução da freqüência cardíaca.

A hipertrofia ventricular esquerda desenvolve-se como processo compensatório ou adaptativo a um estímulo hemodinâmico, representando a sobrecarga de pressão e/ ou volume. A teoria, que melhor explica os padrões de hipertrofia, considera que a resposta ventricular se processa no sentido de manter o estresse parietal ventricular relativamente constante e o volume sistólico adequado.

O exercício físico é um estímulo bem identificado para o desenvolvimento de hipertrofia ventricular esquerda. As alterações estruturais, resultantes do treinamento físico, dependem da natureza, duração e intensidade do exercício. As diversas modalidades esportivas têm sido classificadas, fundamentalmente, em dois grandes grupos: esportes de resistência, nos quais predominam as formas isotônicas ou dinâmicas de exercício, e esportes de força, nos quais predominam as formas isométricas ou estáticas de exercício. Entretanto, raramente, o condicionamento atlético é puramente isotônico ou isométrico, a maioria das atividades físicas envolve componente dinâmico e estático, embora com predomínio de um deles 8 ,20-22.

Outro aspecto relevante é que a sobrecarga hemodinâmica associada ao exercício representa, provavelmente, - mecanismo primário responsável pelas alterações estruturais cardíacas. Em modelos animais ${ }^{23}$, observouse que a hipertrofia miocárdica se relacionou estreitamente com a intensidade da sobrecarga hemodinâmica, enquanto as catecolaminas plasmáticas e cardíacas e a atividade alfa e beta-adrenérgica exerceram efeito mínimo. 
Além do tipo e intensidade do exercício, outros determinantes importante das adaptações estruturais do coração do atleta são a idade, sexo, raça e o componente genético ${ }^{24,25}$. Há consenso na literatura que fatores genéticos devam desempenhar importante papel no desenvolvimento da hipertrofia ventricular em atletas, para justificar as acentuadas diferenças nas alterações cardíacas e na performance atlética, observadas em indivíduos com as mesmas características antropométricas e submetidos ao mesmo nível de treinamento ${ }^{1,24,26}$. Para Pelliccia ${ }^{27}$, os fatores hereditários influenciam as dimensões cardíacas, seja pelo controle genético da resposta hipertrófica ao condicionamento atlético, seja pela predisposição genética para sustentar treinamento físico mais intensivo e atingir níveis mais elevados de performance durante a competição. Recentemente, Montgomery e cols. ${ }^{28}$ estudaram 400 recrutas do exército antes e após um período de 10 semanas de treinamento de força e resistência. Ao final do estudo, observaram aumento da média da massa ventricular esquerda de $167 \mathrm{~g}$ para $197 \mathrm{~g}$ (aumento de $18 \%$ ). Os indivíduos foram divididos em três grupos, de acordo com o polimorfismo do genótipo da enzima de conversão da angiotensina (ECA): inserção homozigótica $(\mathrm{I} / \mathrm{I})$, inserção/deleção heterozigótica (I/D) e deleção homozigótica (D/D). Os três grupos, com o alelo deleção, tiveram o maior aumento da massa ventricular esquerda, sugerindo que níveis aumentados da ECA desempenhem papel importante no desenvolvimento da hipertrofia miocárdica induzida pelo treinamento.

Neste estudo, a avaliação ecocardiográfica da função ventricular esquerda sistólica, representada pela fração de ejeção $(0,61$ a 0,79, média 0,70 00,05$)$ e pelo percentual de encurtamento sistólico da fibra (33 a 49\%, média $40 \% \pm 4$ ), evidenciou valores normais em todos os casos, inclusive significativamente superiores aos do grupo controle

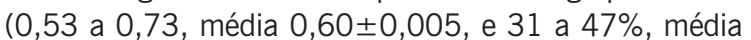
$36,0 \% \pm 4$, respectivamente). Esses achados estão de acordo com os resultados de vários estudos transversais, que compararam grupos de atletas das mais diferentes modalidades esportivas com controles sedentários 3,29-31.

Dois estudos merecem atenção especial. Em atletas praticantes de esportes de resistência e força, observouse que os diversos índices de contratilidade não invasivos utilizados para avaliar a função ventricular esquerda foram normais, apesar do aumento acentuado da cavidade e espessura ventriculares ${ }^{30}$. Outro estudo ${ }^{5}$ avaliou a dimensão ecocardiográfica da cavidade ventricular esquerda em 1.309 atletas de elite, participantes de 38 diferentes modalidades esportivas. De acordo com um nível arbitrário de corte de $60 \mathrm{~mm}$, consideraram que a cavidade ventricular esquerda estava substancialmente aumentada em 185 atletas (14\%). Todos tinham função ventricular esquerda sistólica global dentro dos limites da normalidade e não apresentavam anormalidades da motilidade parietal segmentar. Após seguimento de 1 a 12 anos (média 4,7 anos), os 185 atletas permaneceram assintomáticos e não apresen- taram anomalia da performance cardíaca. Os principais determinantes da dimensão da cavidade ventricular esquerda foram a maior superfície corpórea e a participação em determinados esportes de resistência (ciclismo, esqui de campo e canoagem).

Os resultados da avaliação pela ressonância magnética neste estudo mostraram, igualmente, que, apesar do aumento da massa ventricular esquerda e da espessura parietal, os atletas tinham fração de ejeção normal (57 a $72 \%$, média $63,67 \% \pm 4,53)$. Os achados normais da movimentação regional, analisada pelo tagging, e da mensuração do espessamento regional reforçam a conclusão que não havia prejuízo da força contrátil do coração ou da performance ventricular esquerda. Pluim e cols. $^{32}$ avaliaram a anatomia, função e metabolismo cardíacos pela ressonância magnética e espectroscopia em ciclistas altamente treinados e em indivíduos controles. A massa ventricular esquerda e os volumes diastólicos finais, indexados pela superfície corpórea, foram significantemente maiores nos ciclistas, porém, a fração de ejeção ventricular esquerda, o índice cardíaco e o estresse parietal sistólico não diferiram dos controles. 0 índice fosfocreatina/trifosfato de adenosina foi similar em ambos os grupos. Para os autores, esses achados indicam que a hipertrofia ventricular esquerda induzida pelo exercício físico é apenas adaptação fisiológica.

A avaliação dos diversos índices ecocardiográficos de função diastólica ventricular esquerda - velocidades das ondas $E$ e $A$ do fluxograma mitral, relação $E / A$, tempo de desaceleração da onda $E$ e tempo de relaxamento isovolumétrico - nos atletas deste estudo mostrou valores uniformemente dentro dos limites da normalidade, em condições basais.

Utilizando angiografia com radionuclídeos, Granger e cols. ${ }^{33}$ observaram que atletas não apresentavam alterações no enchimento ventricular esquerdo, embora a massa ventricular esquerda fosse $43 \%$ maior que a do grupo controle sedentário.

Ao contrário da hipertrofia do atleta, a disfunção ventricular diastólica é identificada na grande maioria dos pacientes com hipertensão arterial sistêmica ou miocardiopatia hipertrófica, independente do grau de hipertrofia, da presença de sintomas ou da obstrução da via de saída ${ }^{32}$.

A natureza da textura miocárdica, como a intensidade do eco, pode ser examinada de várias maneiras. A simples codificação pela cor ou a refletividade miocárdica patológica exibe aumento da amplitude do eco associado à fibrose. Por outro lado, na hipertrofia do atleta, a amplitude do eco ou a refletividade miocárdica ultra-sônica situam-se dentro dos limites normais, sugerindo que a hipertrofia fisiológica não se acompanha de fibrose e outras alterações estruturais, observadas em condições patológicas ${ }^{33}$.

Resposta ao exercício - É reconhecido, de longa data, que, em presença de hipertrofia ventricular esquerda, testes de esforço podem apresentar alterações de ST-T típicas de resposta isquêmica pela desproporção ente oferta e consumo 
de oxigênio, mesmo na ausência de doença aterosclerótica coronária obstrutiva. Em pacientes com hipertrofia ventricular esquerda e artérias coronárias normais ao estudo angiográfico, testes ergométricos com resposta isquêmica típica foram relatados em $38 \% 37$ e $58 \% 38$.

No presente estudo, os testes ergométricos não evidenciaram, em nenhum caso, resposta isquêmica ao exercício, nem foram observados sintomas, arritmias, comportamento anormal da freqüência cardíaca ou da função ventricular. Esses resultados indicam que a hipertrofia ventricular esquerda dos atletas não induz à desproporção entre oferta e consumo de oxigênio, mesmo em condições de esforço máximo. Tais achados estão de acordo com os relatados em corredores de longa distância ${ }^{2}$, jogadores de bola-ao-cesto ${ }^{3}$ e futebolistas profissionais $^{29}$, muitos dos quais tinham hipertrofia ventricular esquerda documentada.

O consumo máximo de oxigênio (VO2 max) é o principal indicador dessas adaptações e, provavelmente, o indicador isolado mais objetivo do nível de treinamento físico. Enquanto um adulto jovem normal, não atleta, apresenta VO2 max da ordem de $35 \mathrm{ml} / \mathrm{kg} / \mathrm{min}$, em atletas de resistência, essa variável aumenta significantemente (50\%, em média) ${ }^{22,24,30}$, podendo ultrapassar $70 \mathrm{ml} / \mathrm{kg} / \mathrm{min}^{20,21}$.

A capacidade física dos 30 atletas foi determinada pelo consumo máximo de oxigênio (VO2 max), obtido indiretamente, e seu equivalente em MET. 15 atletas foram sorteados para a submeterem-se a RM, pela medida direta do consumo de 02 . A classificação dos resultados baseou-se na tabela de consumo de 02, elaborada por Barros e cols. ${ }^{19}$, subdividida em quatro categorias: descondicionado, condicionado, bem condicionado e atleta de elite. Os índices alcançados neste trabalho foram de atletas bem condicionados.

Estudos diversos demonstraram que as alterações da massa ventricular esquerda e do tamanho da cavidade ventricular com o treinamento e condicionamento físico

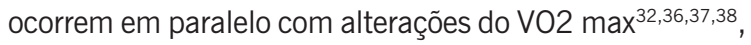
sugerindo, fortemente, que a hipertrofia ventricular se associa com melhor função cardíaca ${ }^{20}$.

Em síntese, os resultados deste estudo evidenciaram que a hipertrofia ventricular esquerda de maratonistas, com excelente nível de treinamento, tem função ventricular esquerda normal, tanto sistólica como diastólica, consumo máximo de oxigênio elevado e não apresentam respostas anômalas ao teste ergométrico.

As razões pelas quais a hipertrofia ventricular esquerda (HVE) dos atletas tem características nitidamente diferentes da hipertrofia associada à hipertensão, miocardiopatia hipertrófica e outras condições patológicas, não estão estabelecidas, porém algumas sugestões podem ser feitas, embasadas em achados clínicos e/ou experimentais.
A primeira e, provavelmente, mais importante é que a sobrecarga de pressão e de volume resultantes do treinamento físico intensivo representa apenas um estímulo hemodinâmico para o desenvolvimento da HVE, sem necessariamente acompanhar-se de alterações neurohumorais, como ocorre nas hipertrofias patológicas. Experimentos em animais ${ }^{23}$ mostraram que a sobrecarga hemodinâmica, associada ao exercício, relaciona-se estreitamente com a hipertrofia miocárdica, enquanto as catecolaminas cardíacas e plasmáticas e a atividade alfa beta-adrenérgica exercem apenas efeito discreto.

Estudos mais recentes, utilizando modelos animais com sobrecarga de volume sem ativação do sistema renina-angiotensina, induziram hipertrofia miocítica, porém não fibrose miocárdica. Assim sendo, o estímulo inicial principal para a hipertrofia miocárdica é estiramento mecânico, enquanto os estímulos que resultam em fibrose são humorais ${ }^{21,22,27}$. Em conseqüência, a hipertrofia do atleta parece restringir-se aos miócitos, sem alterar a matriz extracelular e sem provocar fibrose intersticial1,20,21. Alguns estudos comprovaram, pela codificação da cor ou pela refletividade miocárdica ultra-sônica, que o miocárdio hipertrofiado patologicamente exibe aumento da amplitude do eco associado à fibrose, o que não ocorre na hipertrofia ventricular dos atletas ${ }^{34}$.

Durante o treinamento físico intensivo, o sistema nervoso simpático é ativado, porém, nos períodos de repouso, muito mais prolongados em cada dia, ocorre preponderância vagal $^{21,22}$, que, provavelmente, contrabalança os efeitos deletérios das catecolaminas no miocárdio, bem como a ativação de outros sistemas neuro-hormonais pelo sistema nervoso simpático.

O aumento do número de mitocôndrias, a neoformação capilar, a atividade normal da ATPase da miosina observados no miocárdio hipertrofiado pelo treinamento físico, impedem a desproporção entre oferta e consumo de oxigênio e a ocorrência de isquemia, ao contrário do observado nas hipertrofias patológicas ${ }^{21,22}$.

Os resultados do presente estudo, num grupo de maratonistas em período de atividade esportiva plena, permitem concluir que a hipertrofia ventricular esquerda observada nesses atletas, avaliada por métodos não invasivos, representa resposta adaptativa ao treinamento físico intensivo e prolongado, com características puramente fisiológicas.

\section{Agradecimentos}

Aos Drs. Daniel J. Daher, Almir S. Ferraz, Mohamed Saleh, Romeu Sérgio Meneghelo, Sérgio C. Pontes e Hélio J. Schwartz, pela colaboração prestada. 


\section{REFERÊNCIAS}

1. Rost R. Athletics and the heart. Chicago, Year Book Medical Publishers, 1986.

2. Raskoff WJ, Goldman S, Cohn K. The athletic heart: prevalence and physiological significance of left ventricular enlargement in distance runners. JAMA 1976; 236: 158-62.

3. Roeske WR, O'Rourke RA, Klein A et al. Noninvasive evaluation of ventricular hypertrophy in professional athletes. Circulation 1976; 53: 286-92.

4. Morganroth J, Maron BJ, Henry WL. Comparative left ventricular dimensions in trained athletes. Ann Intern Med 1975; 82: 521-4.

5. Pelliccia A, Culasso F, Di Paolo FM, Maron BJ. Physiologic left ventricular cavity dilatation in elite athletes. Ann Intern Med 1999; 130: 23-31

6. Ccann GP, Muir DF, Hills WS. Athletic left ventricular hypertrophy: longterm studies are required. [Editorial]. Eur Heart J 2000; 21: 351-3.

7. Bevegard S, Holmgren A, Johnson B. Circulatory studies in well-trained athletes at rest and during exercise, with special reference to stroke volume and influence of body position. Acta Physiol Scand 1963; 57: 26-38.

8. Frick MH, Konttinen A, Sarajas HSS. Effects of physical training on circulation at rest and during exercise. Am J Cardiol 1963; 12: 142-7.

9. Saltin B, Astran PO. Maximal oxygen uptake in athletes. J Appl Physiol 1967; 23:353-8.

10. Scheur J, Pehpargkul S, Bhan A. Experimental observations on the effects of physical training upon intrinsic physiology and biochemistry. Am J Cardiol 1974; 33: 744-51

11. Blomqvist G, Saltin B. Cardiovascular adaptations to physical training. Annu Rev Physical 1983; 45: 169-89.

12. Maron BJ. Sudden cardiac death due to hypertrophic cardiomyopathy in young athletes. In: Thompson PD, ed. Exercise and Sports Cardiology. New York: McGraw-Hill 2001: 189-210.

13. Schwartz HJ, Abraão HD. Eletrocardiografia - vectocardiografia. In: Ghorayeb N, Meneghelo RS (eds). Métodos Diagnósticos em Cardiologia, Rio de Janeiro: Atheneu 1997: 2-8.

14. Bruce RA. Exercise testing of patients with coronary heart disease. Ann Clin Res 1971; 3: 323.

15. Weiman AE. Appendix A, normal cross-sectional echocardiographic measurements. In: Principles and Practice of Echocardiogram. 2nd Ed. Pennsylvania: Lea \& Febiger 1994: 1288-98.

16. Martin ET, Fuisz AR, Pohost GM. Imaging cardiac structure and pump function. Cardiol Clin 1998; 16: 135-60.

17. Sayad D, Willet DW, Chwialkowski M et al. Noninvasive quantitation of regional left ventricular function using gradient-echo MRI with myocardial tagging: interstudy reproducibility. Am J Cardiol 1995; 76 : 985-8.

18. Simonson E. Differentiation Between Normal and Abnormal Eletrocardiography. St Louis: CV Mosby 1961: 150-5.

19. Barros Neto TL, César MC, Tambeiro VL. Avaliação da aptidão física cardiorrespiratória. In: Ghorayeb N, Barros T, eds. O Exercício: Preparação Fisiológica, Avaliação Médica, Aspectos Especiais e Preventivos. Rio de Janeiro: Atheneu 1999: 15-24.

20. Ghorayeb N, Batlouni M. Hipertrofia ventricular: mecanismos envolvidos na indução e possibilidades de regressão. Rev Soc Cardiol Est São Paulo: 1998; 8: 298-301.

21. Batlouni M. Coração de atleta. In: Ghorayeb N, Barros Neto TL, eds. O Exercício: Preparação Fisiológica, Avaliação Médica, Aspectos Especiais e Preventivos. Rio de Janeiro: Atheneu 1999: 147-72.
22. Barros Neto TL. Hipertrofia ventricular secundária ao exercício físico. Rev Soc Cardiol Est São Paulo 1994; 4: 376-81.

23. Cooper IV G, Kent RL, Uboh CE, Thompson EW, Marino TA. Hemodynamic versus adrenergic control of cat right ventricular hypertrophy. J Clin Invest 1985; 75: 1403-14.

24. Maron BJ. Structural features of the athletes heart as defined by echocardiography. J Am Coll Cardiol 1986; 7: 190-203.

25. Douglas PS, O'Toole ML, Katz SE. Left ventricular hypertrophy in athletes. Am J Cardiol 1997; 80: 1384-90

26. Osborne G, Wolfe LA, Burggraf GW et al. Relationships between cardiac dimensions, anthropometric characteristics and maximal aerobic power in young men. Int J Sports Med 1992; 13: 219-24.

27. Pelliccia A. Determinants of morphologic cardiac adaptation in elite athletes: the role of athletic training and constitutional factors. Int J Sports Med 1996; 17 (suppl.): 157-63.

28. Montgomery HE, Clarkson P, Dollery CM et al Association of angiotensinconverting enzymes gene I/D polymorphism with change in left ventricular mass in response to physical training. Circulation 1997; 96: 741-7.

29. Batlouni M, Abrão HD, Ghorayeb N, Gizzi LC, Zorzo D, Sauaia N. Alterações eletrocardiográficas em atletas profissionais: I. Sobrecargas ventriculares. Arq Bras Cardiol 1980; 35: 95-110.

30. Pelliccia A, Maron BJ, Spataro A et al. The upper limit of physiologic cardiac hypertrophy in highly trained elite athletes. N Engl J Med 1991; 324: 295-301

31. Colan SD, Sanders SP, Borow KM. Physiological hypertrophy: effects on left ventricular systolic mechanics in athletes. J Am Coll Cardiol 1987; 9: 776-9.

32. Pluim BM, Zwinderman AH, Laarse A et al. The athlete's heart: a meta-analysis of cardiac structure and function. Circulation 1999; 100: 336-44.

33. Granger CB, Karimeddini MK, Smith VE et al. Rapid ventricular filling in left ventricular hypertrophy. Physiologic hypertrophy. J Am Coll Cardiol 1985; 5: 862-8.

34. Lewis JF, Spirito P, Pelliccia A, Maron BJ. Usefulness of Doppler echocardiographic assessment of diastolic filling in distinguishing "athlete's heart" from hypertrophic cardiomyopathy. Br Heart J 1992; 68: 296-300.

35. Lattanzi F, Di Bello V, Picano E et al.Normal ultrasonic myocardial reflectivity in athletes with increased left ventricular mass. Circulation 1992; 85: 1828-34

36. Batlouni M. Hipertrofia cardíaca: fatores determinantes e mecanismos moleculares. Arq Bras Cardiol 1995; 65: 533-9.

37. Ehsani AA, Hagberg JM, Hickson RC. Rapid changes in left ventricular dimension and mass in response to physical conditioning deconditioning. Am J Cardiol 1978; 42: 52-6.

38. Harris CN, Aronow WS, Parker DP et al. Treadmill stress test in left ventricular hypertrophy. Chest 1973; 63: 353-7.

39. Roitman D, Jones WB, Sheffield LT. Comparison of submaximal exercise test with coronary cineangiogram. Ann Intern Med 1970; 72: 641-7.

40. Grossman W. Cardiac hypertrophy: useful adaptation or pathologic process? Am J Med 1980; 69: 576-84.

41. Wikman-Coffel TJ, Parmley WW, Mason DT. The cardiac hypertrophy process. Circ Res 1980; 45: 697-707.

42. Shapiro LM. Conseqüências morfológicas do treinamento sistemático. In: Maron BJ. O Coração de Atleta e a Doença Cardiovascular. Rio de Janeiro: Interlivros Edições Ltda. Série Clínicas Cardiológicas 1997; 3: 373-9. 